\title{
Normal Subgroup of Product of Groups
}

\author{
Hiroyuki Okazaki \\ Shinshu University \\ Nagano, Japan
}

\author{
Kenichi Arai \\ Shinshu University \\ Nagano, Japan
}

\author{
Yasunari Shidama \\ Shinshu University \\ Nagano, Japan
}

Summary. In [6] it was formalized that the direct product of a family of groups gives a new group. In this article, we formalize that for all $j \in I$, the group $G=\prod_{i \in I} G_{i}$ has a normal subgroup isomorphic to $G_{j}$. Moreover, we show some relations between a family of groups and its direct product.

MML identifier: GROUP_12, version: $\underline{7.11 .07 \quad 4.156 .1112}$

The papers [2], [4], [5], [3], [8], [9], [7], [10], [11], [6], [1], [13], and [12] provide the terminology and notation for this paper.

\section{Normal Subgroup of Product of Groups}

Let $I$ be a non empty set, let $F$ be a group-like multiplicative magma family of $I$, and let $i$ be an element of $I$. Note that $F(i)$ is group-like.

Let $I$ be a non empty set, let $F$ be an associative multiplicative magma family of $I$, and let $i$ be an element of $I$. Observe that $F(i)$ is associative.

Let $I$ be a non empty set, let $F$ be a commutative multiplicative magma family of $I$, and let $i$ be an element of $I$. Note that $F(i)$ is commutative.

In the sequel $I$ is a non empty set, $F$ is an associative group-like multiplicative magma family of $I$, and $i, j$ are elements of $I$.

We now state the proposition

(1) Let $x$ be a function and $g$ be an element of $F(i)$. Then dom $x=I$ and $x(i)=g$ and for every element $j$ of $I$ such that $j \neq i$ holds $x(j)=\mathbf{1}_{F(j)}$ if and only if $x=\mathbf{1}_{\prod F}+\cdot(i, g)$.

Let $I$ be a non empty set, let $F$ be an associative group-like multiplicative magma family of $I$, and let $i$ be an element of $I$. The functor $\operatorname{ProjSet}(F, i)$ yields a subset of $\prod F$ and is defined by: 
(Def. 1) For every set $x$ holds $x \in \operatorname{ProjSet}(F, i)$ iff there exists an element $g$ of $F(i)$ such that $x=\mathbf{1}_{\prod F}+\cdot(i, g)$.

Let $I$ be a non empty set, let $F$ be an associative group-like multiplicative magma family of $I$, and let $i$ be an element of $I$. Observe that $\operatorname{ProjSet}(F, i)$ is non empty.

Next we state several propositions:

(2) Let $x_{0}$ be a set. Then $x_{0} \in \operatorname{ProjSet}(F, i)$ if and only if there exists a function $x$ and there exists an element $g$ of $F(i)$ such that $x=x_{0}$ and $\operatorname{dom} x=I$ and $x(i)=g$ and for every element $j$ of $I$ such that $j \neq i$ holds $x(j)=\mathbf{1}_{F(j)}$.

(3) Let $g_{1}, g_{2}$ be elements of $\prod F$ and $z_{1}, z_{2}$ be elements of $F(i)$. If $g_{1}=$

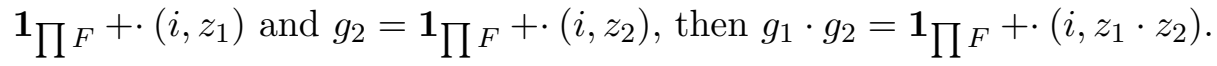

(4) For every element $g_{1}$ of $\prod F$ and for every element $z_{1}$ of $F(i)$ such that $g_{1}=\mathbf{1}_{\prod F}+\cdot\left(i, z_{1}\right)$ holds $g_{1}^{-1}=\mathbf{1}_{\prod F}+\cdot\left(i, z_{1}{ }^{-1}\right)$.

(5) For all elements $g_{1}, g_{2}$ of $\prod F$ such that $g_{1}, g_{2} \in \operatorname{ProjSet}(F, i)$ holds $g_{1} \cdot g_{2} \in \operatorname{ProjSet}(F, i)$.

(6) For every element $g$ of $\prod F$ such that $g \in \operatorname{ProjSet}(F, i)$ holds $g^{-1} \in$ $\operatorname{ProjSet}(F, i)$.

Let $I$ be a non empty set, let $F$ be an associative group-like multiplicative magma family of $I$, and let $i$ be an element of $I$. The functor $\operatorname{ProjGroup}(F, i)$ yields a strict subgroup of $\prod F$ and is defined as follows:

(Def. 2) The carrier of $\operatorname{ProjGroup}(F, i)=\operatorname{ProjSet}(F, i)$.

Let us consider $I, F, i$. The functor $1 \operatorname{ProdHom}(F, i)$ yielding a homomorphism from $F(i)$ to $\operatorname{Proj} \operatorname{Group}(F, i)$ is defined as follows:

(Def. 3) For every element $x$ of $F(i)$ holds $(1 \operatorname{ProdHom}(F, i))(x)=\mathbf{1}_{\prod F}+\cdot(i, x)$.

Let us consider $I, F, i$. Note that $1 \operatorname{ProdHom}(F, i)$ is bijective.

Let us consider $I, F, i$. One can check that $\operatorname{ProjGroup}(F, i)$ is normal.

One can prove the following proposition

(7) For all elements $x, y$ of $\prod F$ such that $i \neq j$ and $x \in \operatorname{Proj} \operatorname{Group}(F, i)$ and $y \in \operatorname{ProjGroup}(F, j)$ holds $x \cdot y=y \cdot x$.

\section{Product of Subgroups of a Group}

In the sequel $n$ denotes a non empty natural number.

One can prove the following propositions:

(8) Let $F$ be an associative group-like multiplicative magma family of $\operatorname{Seg} n$, $J$ be a natural number, and $G_{1}$ be a group. Suppose $1 \leq J \leq n$ and $G_{1}=F(J)$. Let $x$ be an element of $\prod F$ and $s$ be a finite sequence of elements of $\prod F$. Suppose len $s<J$ and for every element $k$ of $\operatorname{Seg} n$ 
such that $k \in \operatorname{dom} s$ holds $s(k) \in \operatorname{ProjGroup}(F, k)$ and $x=\prod s$. Then $x(J)=\mathbf{1}_{\left(G_{1}\right)}$.

(9) Let $F$ be an associative group-like multiplicative magma family of Seg $n, x$ be an element of $\prod F$, and $s$ be a finite sequence of elements of $\prod F$. Suppose len $s=n$ and for every element $k$ of $\operatorname{Seg} n$ holds $s(k) \in \operatorname{ProjGroup}(F, k)$ and $x=\prod s$. Let $i$ be a natural number. Suppose $1 \leq i \leq n$. Then there exists an element $s_{1}$ of $\prod F$ such that $s_{1}=s(i)$ and $x(i)=s_{1}(i)$.

(10) Let $F$ be an associative group-like multiplicative magma family of $\operatorname{Seg} n$, $x$ be an element of $\prod F$, and $s, t$ be finite sequences of elements of $\prod F$. Suppose that

(i) $\operatorname{len} s=n$,

(ii) for every element $k$ of $\operatorname{Seg} n$ holds $s(k) \in \operatorname{ProjGroup}(F, k)$,

(iii) $x=\prod s$,

(iv) len $t=n$,

(v) for every element $k$ of $\operatorname{Seg} n$ holds $t(k) \in \operatorname{ProjGroup}(F, k)$, and

(vi) $x=\prod t$.

Then $s=t$.

(11) Let $F$ be an associative group-like multiplicative magma family of $\operatorname{Seg} n$ and $x$ be an element of $\prod F$. Then there exists a finite sequence $s$ of elements of $\prod F$ such that len $s=n$ and for every element $k$ of $\operatorname{Seg} n$ holds $s(k) \in \operatorname{ProjGroup}(F, k)$ and $x=\prod s$.

(12) Let $G$ be a commutative group and $F$ be an associative group-like multiplicative magma family of $\operatorname{Seg} n$. Suppose that

(i) for every element $i$ of $\operatorname{Seg} n$ holds $F(i)$ is a subgroup of $G$,

(ii) for every element $x$ of $G$ there exists a finite sequence $s$ of elements of $G$ such that len $s=n$ and for every element $k$ of $\operatorname{Seg} n$ holds $s(k) \in F(k)$ and $x=\prod s$, and

(iii) for all finite sequences $s, t$ of elements of $G$ such that len $s=n$ and for every element $k$ of $\operatorname{Seg} n$ holds $s(k) \in F(k)$ and len $t=n$ and for every element $k$ of Seg $n$ holds $t(k) \in F(k)$ and $\prod s=\prod t$ holds $s=t$.

Then there exists a homomorphism $f$ from $\prod F$ to $G$ such that

(iv) $f$ is bijective, and

(v) for every element $x$ of $\prod F$ there exists a finite sequence $s$ of elements of $G$ such that len $s=n$ and for every element $k$ of $\operatorname{Seg} n$ holds $s(k) \in F(k)$ and $s=x$ and $f(x)=\prod s$.

(13) Let $G, F$ be associative commutative group-like multiplicative magma families of $\operatorname{Seg} n$. Suppose that for every element $k$ of $\operatorname{Seg} n$ holds $F(k)=$ $\operatorname{ProjGroup}(G, k)$. Then there exists a homomorphism $f$ from $\prod F$ to $\prod G$ such that

(i) $f$ is bijective, and 
(ii) for every element $x$ of $\prod F$ there exists a finite sequence $s$ of elements of $\prod G$ such that len $s=n$ and for every element $k$ of $\operatorname{Seg} n$ holds $s(k) \in F(k)$ and $s=x$ and $f(x)=\prod s$.

\section{REFERENCES}

[1] Grzegorz Bancerek. The fundamental properties of natural numbers. Formalized Mathematics, 1(1):41-46, 1990.

[2] Grzegorz Bancerek and Krzysztof Hryniewiecki. Segments of natural numbers and finite sequences. Formalized Mathematics, 1(1):107-114, 1990.

[3] Grzegorz Bancerek and Andrzej Trybulec. Miscellaneous facts about functions. Formalized Mathematics, 5(4):485-492, 1996.

[4] Czesław Byliński. Functions and their basic properties. Formalized Mathematics, 1(1):5565, 1990.

[5] Czesław Byliński. Functions from a set to a set. Formalized Mathematics, 1(1):153-164, 1990.

[6] Artur Korniłowicz. The product of the families of the groups. Formalized Mathematics, $7(\mathbf{1}): 127-134,1998$.

[7] Wojciech A. Trybulec. Classes of conjugation. Normal subgroups. Formalized Mathematics, 1(5):955-962, 1990.

[8] Wojciech A. Trybulec. Groups. Formalized Mathematics, 1(5):821-827, 1990.

[9] Wojciech A. Trybulec. Subgroup and cosets of subgroups. Formalized Mathematics, 1(5):855-864, 1990.

[10] Wojciech A. Trybulec. Lattice of subgroups of a group. Frattini subgroup. Formalized Mathematics, 2(1):41-47, 1991.

[11] Wojciech A. Trybulec and Michał J. Trybulec. Homomorphisms and isomorphisms of groups. Quotient group. Formalized Mathematics, 2(4):573-578, 1991.

[12] Zinaida Trybulec. Properties of subsets. Formalized Mathematics, 1(1):67-71, 1990.

[13] Edmund Woronowicz. Relations and their basic properties. Formalized Mathematics, 1(1):73-83, 1990. 